Kansas State University Libraries

New Prairie Press

Conference on Applied Statistics in Agriculture

2005 - 17th Annual Conference Proceedings

\title{
DESIGN AND ANALYSIS OF BIOLOGICAL ASSAYS OF MIXTURES
}

Nancy Ferry

Bruce H. Stanley

Gregory Armel

Follow this and additional works at: https://newprairiepress.org/agstatconference

Part of the Agriculture Commons, and the Applied Statistics Commons

\section{(c) (1) $\Theta$}

This work is licensed under a Creative Commons Attribution-Noncommercial-No Derivative Works 4.0 License.

\section{Recommended Citation}

Ferry, Nancy; Stanley, Bruce H.; and Armel, Gregory (2005). "DESIGN AND ANALYSIS OF BIOLOGICAL ASSAYS OF MIXTURES," Conference on Applied Statistics in Agriculture. https://doi.org/10.4148/

2475-7772.1133

This is brought to you for free and open access by the Conferences at New Prairie Press. It has been accepted for inclusion in Conference on Applied Statistics in Agriculture by an authorized administrator of New Prairie Press. For more information, please contact cads@k-state.edu. 


\title{
DESIGN AND ANALYSIS OF BIOLOGICAL ASSAYS OF MIXTURES
}

\author{
Nancy Ferry, Dr. Bruce H. Stanley and Dr. Gregory Armel \\ DuPont Crop Protection Products
}

Newark, DE 19714

\begin{abstract}
The simultaneous activity of multiple stimuli can be difficult to analyze, particularly on biological systems. However, these analyses are becoming increasingly important in drug or pesticide formulation for efficacy. This article will review techniques for the design and analysis of bioassays of mixtures. The two major techniques that will be reviewed are based upon the concepts of response and potency. Particular emphasis will be placed upon measuring levels of synergy, i.e., when the activity is greater than the sum of its parts, and antagonism, i.e., when the activity is less than would be expected. Theoretical examples will be given to demonstrate the application of each technique. The method based on potency was used in the design and analysis of Dr. Gregory Armel's study of the differential response of atrazine mixtures with bleaching herbicides that target different sites in carotenoid biosynthesis. Examples from this mixture study are presented.
\end{abstract}

Keywords: Mixture, isobole method, synergy, antagonism, potency

\section{Introduction}

Crop protection products (e.g., herbicides, insecticides, and fungicides) help control the thousands of weed species, harmful insects and numerous plant diseases that afflict crops. There are many reasons why crop protection products are mixed. These include:

- Farmers often deal with multiple pests attacking their crops. One crop protection product that controls a broad spectrum of pests is simpler to use and easier to market.

- A combination of crop protection products may increase the duration of control (e.g., a fast acting product combined with a slower acting persistent partner.) The combination of two products may result in synergy, with increased potency. Synergy can result in benefits to the environment, the farmer, and the producer, as lower concentrations of each of the mixture partners can be used, yet still yield effective control.

- Conversely, the combination of two products may result in antagonism, with two products being mixed together and giving less than the expected level of activity. This antagonism can be valuable as safening, reducing crop response to a crop protection product.

- Crop pests are dynamic populations. The 'survival of the fittest' dynamic can lead to reduced susceptibility of the pest population to a crop protection product. Resistance to crop production products is a scenario that negatively impacts both farmers and crop protection companies. One way to help manage this 'resistance' is to apply products with different modes of action. Mixtures of two products with different modes of action are attractive for this reason. 
- Mixtures can also extend the patent life of a partner in the mixture and help a company defend market position.

Mixing two crop protection products can result in one of three types of activity. Independent or additive activity occurs when a product behaves the same regardless of the presence of another product; there is zero interaction. Synergy occurs when products mixed together give greater than the expected level of activity; the products are working together. Antagonism occurs when products mixed together give less than the expected level of activity; the products are working against each other.

Two of the typical methods for assessing joint action are Colby's Equation (Colby, 1967) and the Isobole method (Berenbaum, 1989). Colby's Equation works with responses and the Isobologram method works with potency. These methods are applicable to mixtures of more than two products, however the discussion here is limited to the mixtures of two products.

\section{Data and Methodology}

\subsection{Colby's Equation}

To use the Colby approach to assessing synergy or antagonism in mixtures, the component partners in the mixture must be tested singly at their component concentration in the same study with the mixture. This historical approach has the appeal of being easy to calculate and interpret. The observed results for the mixture are compared with those expected from the Colby Equation (Colby, 1967). Note that this equation is derived from the probability definition of independence. This approach works with the responses, given a particular rate (i.e., works with the y's at a given x's).

Assuming responses range from 0 to 100 percent pest reduction:

$\mathrm{X}=$ Observed result from compound $\mathrm{A}$ at $\mathrm{p}$ grams ai/ha

$\mathrm{Y}=$ Observed result from compound $\mathrm{B}$ at $\mathrm{q}$ grams ai/ha

$\mathrm{E}=$ Expected result for mixture of $\mathrm{A}$ and $\mathrm{B}$ at $(\mathrm{p}+\mathrm{q}) \mathrm{g}$ ai/ha if there is no synergy or antagonism

$E=X+(100-X)(Y / 100)$

$=\mathbf{X}+\mathbf{Y}-\mathbf{X}^{*} \mathbf{Y} / \mathbf{1 0 0}$

\section{Conclusions:}

If observed value is greater than expected result (Obs $>\mathrm{E})$ : synergy

If observed value is less than expected result (Obs $<\mathrm{E})$ : antagonism

The biologists must determine what amount of difference between observed and expected is biologically meaningful. This method can be generalized to more than two partners,

To generalize to a third mixing partner, Z:

$$
E=\mathrm{X}+\mathrm{Y}+\mathrm{Z}-\frac{(\mathrm{X} * \mathrm{Y})+(\mathrm{X} * \mathrm{Z})+(\mathrm{Y} * \mathrm{Z})}{100}+\frac{\mathrm{X}^{*} \mathrm{Y} * \mathrm{Z}}{100^{3-1}}
$$




\subsection{Isobole Method}

M. C. Berenbaum’s “What is Synergy” article, published in 1989, gives a thorough treatment of methods for assessing interactions between biologically active agents. He reviews the literature claiming synergy of biologically active agents and the various approaches for analyzing interaction (a total of 564 references are given). He exposes the weaknesses and lack of generality of many of the approaches and recommends the isobole method as a "generally valid procedure for analyzing interactions between agents irrespective of their mechanisms of action or the nature of their dose-response relations” (Berenbaum, 1989, p99). Berenbaum presents a comprehensive proof of the general validity of the isobole method. The isobole method is used to distinguish between the expected increased effect of a combination of active agents and a true interaction (i.e., synergy or antagonism).

The isobole method is based on the dose response of the compounds in a mixture and uses 'isoeffective' or equally effective doses for each of the compounds to build an isobole graph. Dose response bioassays estimate the biological activity of a test substance by means of the reaction that follows its application to living matter. Increasing concentrations of the test substance are applied to biological subjects. The subject's response is a measure of some characteristic of the subject which indicates the biological activity of the stimulus.

The relationship between dose and response is used to calculate the ECx, the expected concentration of a sample predicted to elicit an $\mathrm{X} \%$ response. For example, the $\mathrm{EC}_{50}$ is the concentration of a compound predicted to elicit a 50\% response. A well-designed dose response will yield responses ranging from very little response (e.g., 20\% control) to very high response (e.g., 80\% control). These ECx's are a measure of the potency of a compound. In crop protection studies, dose response assays effectively characterize the biological activity of a compound, the rate at which highest effect is achieved, the break rate (i.e., the rate where pest control begins to drop) and the rate at which effect is lost (i.e., a steep vs shallow dose response curve). Much more information is gained by a dose response for a compound than by a single rate.

The isobole method works with the potency of a mixture and its individual components, the rate predicted to give a specific level of response (works with the $\mathrm{x}$ that gives a specified $\mathrm{y}$ ). It is important that one not extrapolate outside the range of responses. For example, if the maximum response obtained is only $30 \%$ control, do not build an isobole graph using the $\mathrm{EC}_{50}$, rather use an $\mathrm{ECx}$ within the range of responses, for example an $\mathrm{EC}_{20}$. Using the isobole method, interaction is detected when the effect of a combination of agents differs from that expected from their individual dose response curves.

The underlying assumption of the isobole method is that:

$$
\frac{d_{A}}{D_{A}}+\frac{d_{B}}{D_{B}}=s \quad \text { where if } s=\left\{\begin{array}{lll}
<1 & \text { implies } & \text { synergy } \\
1 & \text { implies } & \text { independent action } \\
>1 & \text { implies } & \text { antagonism }
\end{array}\right.
$$

Where:

$\mathrm{D}_{\mathrm{A}}=\quad \mathrm{EC}_{\mathrm{X}}$ for pure compound $\mathrm{A}$ 
$\mathrm{D}_{\mathrm{B}}=\mathrm{EC}_{\mathrm{X}}$ for pure compound $\mathrm{B}$

$\mathrm{d}_{\mathrm{A}}=$ amount of compound $\mathrm{A}$ in the $\mathrm{EC}_{\mathrm{X}}$ of the mixture

$\mathrm{d}_{\mathrm{B}}=$ amount of compound $\mathrm{B}$ in the $\mathrm{EC}_{\mathrm{X}}$ of the mixture

The sum of the relative potencies of the components determine the overall effect. If the sum is one, then there is independent action in the mixture, just what would be expected if the presence of the other partner does not affect the activity of the first partner, and vice versa.

\section{Synergy:}

When agents in combination are more effective than expected from their dose response curves (synergy), smaller amounts are needed to produce the effect under consideration. So $\mathrm{d}_{\mathrm{A}}$ and $d_{B}$ are reduced, while $D_{A}$ and $D_{B}$ are the doses of the agents being used alone (Berenbaum, 1989).

$$
\frac{d_{A}}{D_{A}}+\frac{d_{B}}{D_{B}}<1
$$

If there is synergy, s will be less than 1 , as it takes less of both mixture partners, when used together to yield that same response as each component used separately. Compounds mixed together give greater than expected level of activity.

\section{Antagonism:}

Conversely, when agents in combination are less effective than expected (antagonism), larger amounts are needed to produce the effect under consideration. So $\mathrm{d}_{\mathrm{A}}$ and $\mathrm{d}_{\mathrm{B}}$ are increased, while $\mathrm{D}_{\mathrm{A}}$ and $\mathrm{D}_{\mathrm{B}}$ are the doses of the agents being used alone (Berenbaum, 1989).

$$
\frac{d_{A}}{D_{A}}+\frac{d_{B}}{D_{B}}>1
$$

If there is antagonism, $\mathrm{s}$ will be greater than 1 , as it takes more of both mixture partners to yield a level of activity. Compounds are working against each other. The isobole method is useful for designing mixture studies to efficiently assess a mixture space (i.e., all possible ratios of a mixture of two compounds).

\subsection{Visualization of an Isobologram}

The visualization of an isobole graph, hereafter called an isobologram, helps communicate results to biologists. A visualization of a two-way mixture can be based on any $\mathrm{EC}_{\mathrm{X}}$ of interest. The $\mathrm{EC}_{50}$ is the most stable estimate, assuming a full dose response was generated. For each pure compound and for each mixture, fit the dose response relationship and estimate the expected dose (ie., $\mathrm{EC}_{\mathrm{X}}$ ) that will give the desired percentage response (i.e., $\mathrm{X} \%$ ). Calculate the confidence intervals (usually 95\%) associated with the expected dose eliciting the desired percent response.

To create an isobologram, a graph is created with the two axes being the dose axes of the individual compounds. An independent action line is drawn connecting the two isoeffective doses of the individual compounds (i.e., the $\mathrm{EC}_{50}$ for compound $\mathrm{A}$ and the $\mathrm{EC}_{50}$ for compound 
B). If there is no interaction, it is expected that the independent action line will be a straight line (Berenbaum, 1989). Every point on the line represents a ratio of compound A and compound B that together will give 50\% control, assuming independent action (i.e., no synergy or antagonism).

For the mixtures, separate the amounts of compound A and B from the expected doses (e.g., $\mathrm{EC}_{50} \mathrm{~s}$ ) and from the lower and upper confidence intervals into the amounts of compounds $\mathrm{A}$ and $\mathrm{B}$ required in the mixture to control $\mathrm{x} \%$ of the pest, according to following formulas.

$$
\begin{aligned}
& d_{A_{f g h}}=\left(\frac{1}{1+R_{f}}\right) E C_{X_{f g h}} \\
& d_{B_{f g h}}=\left(\frac{R_{f}}{1+R_{f}}\right) E C_{X_{f g h}}=E C_{X_{f g h}}-d_{A_{f g h}}=R_{f} \cdot d_{A_{f g h}}
\end{aligned}
$$

Where:

$\mathrm{R}_{\mathrm{f}}=\mathrm{f}_{\text {th }}$ ratio of mixture of $\mathrm{A}$ and $\mathrm{B}\left(\mathrm{i} . \mathrm{e} ., 1 \mathrm{~A}:\left(\mathrm{R}_{\mathrm{f}}\right) * \mathrm{~B}\right)$

$\mathrm{f}=\quad$ ratio identifier $(\mathrm{f}=1, \ldots \mathrm{n})$

$\mathrm{g}=$ percentage response identifier (e.g., 20, 50 or 80 )

$\mathrm{h}=\quad$ index for curve (i.e., fitted value, lower and upper confidence intervals)

$\mathrm{d}_{\mathrm{A}}=$ amount of compound $\mathrm{A}$ in the $\mathrm{EC}_{\mathrm{X}}$ of the mixture

$\mathrm{d} \mathrm{B}=$ amount of compound $\mathrm{B}$ in the $\mathrm{EC}_{\mathrm{X}}$ of the mixture

A mixture line is plotted showing the actual amounts of compounds A and B required in the mixture to cause $50 \%$ control of the pest. The mixture data points are plotted by parsing out each mixture's $\mathrm{EC}_{50}$ into its compound $\mathrm{A}$ and compound $\mathrm{B}$ components, based on each ratio. Confidence intervals for the mixture data points are calculated by parsing out the mixture $\mathrm{EC}_{50}$ confidence intervals. The confidence intervals around the mixture line are used to assess synergy or antagonism. If the confidence interval overlaps the independent action line, there is not enough evidence to determine synergy or antagonism. If the upper confidence interval for the mixture line falls under the independent action line, synergy is indicated. If the lower confidence interval for the mixture line falls above the independent action line, antagonism is indicated.

Two hypothetical isobolograms are shown in figures 1 and 2. Figure 1 shows the isobologram for three fixed ratio mixtures. Figure 2 demonstrates how different ratios of the same components can results in assessments of synergy and possible antagonism. This points to the need to investigate your mixture space.

\subsection{Designing a Mixture Study using Isoboles}

The isobole method can be used to investigate the mixture space (i.e., all possible ratios for a mixture). In the same way that theory of Design of Experiments can guide efficient 
identification of a combination of parameters to optimize a response, designing mixture experiments using the isobole method can provide efficient exploration of the mixture space.

The isobole method was used to design a mixture study for Dr. Gregory Armel. Dr. Gregory Armel wanted to assess the differential response of mixtures of the PSII inhibitor Atrazine with bleaching herbicides that target different sites in carotenoid biosynthesis. The objective of this study was to determine whether these mixtures offer synergistic herbicidal activity.

Since the isobole method is based on potency of the mixture components, generating a design requires a good estimate of potency for each of the pure compounds. Sometimes no new data needs to be generated, as the researcher has confidence in the reliability of a known potency for a particular compound. If this is not the case, a pilot test should be run, with a full range of doses, in order to get reliable estimates for the $\mathrm{EC}_{20}, \mathrm{EC}_{50}$ and $\mathrm{EC}_{80}$. This pilot should be conducted under similar conditions that will be used for assessing the mixtures.

One should collect from the biologist the number of mixtures he is willing to test and any limitations on the possible ratios. The isobole method can be used to suggest ratios for testing that are evenly spaced across the mixture space. Dr. Armel wanted to examine mixtures of 5 herbicides with Atrazine to assess herbicidal activity. Dr, Armel ran a pilot study generating reliable estimates of potency for all the individual compounds. Visual assessment of percent control was the response measured in the pilot study on the pure compounds. All of the compounds were tested on five different weed species. Species used were red morningglory (Ipomoea coccinea), common cocklebur (Xanthium strumarium), broadleaf signalgrass (Brachiaria platyphylla), giant foxtail (Setaria faberi), and yellow nutsedge (Cyperus esculentus).

Figure 3 shows dose response curves fit for the pilot test results for two of the herbicidal compounds, CPTA, lycopene cyclase (LPC) (Fedtke et al. 2001) and Atrazine, a photosystem II inhibitor. A dose response curve was fit to the data and ECx estimates calculated using the loglogistic tolerance distribution parameters, using nonlinear least squares, as shown in the SAS code below:

proc nlin; model response $=\left(100 *\right.$ Rate $\left.^{* *} \mathrm{~B}\right) /((\mathrm{R} 50 * * \mathrm{~B})+($ Rate**B $))$; parameters $\mathrm{R} 50=20 \mathrm{~B}=1$;

Table 1 shows the accompanying data tables with average percent control responses and ECx estimates for the two compounds in figure 3. Using the potency estimates for the individual compounds, with no limitations on the mixture space, mixture ratios that evenly split the 'mixture space' were calculated for the five mixtures of herbicides with Atrazine. The following formula (8) is used to calculate which ratios should be tested that evenly divide mixture space. Note that the subscript used with the ratios is different from the subscript used section 2.3.

Formula to calculate $\mathrm{R}_{\mathrm{i}}$, where the $\mathrm{i}_{\text {th }}$ ratio is $1 \mathrm{~A}:\left(\mathrm{R}_{\mathrm{i}}\right) * \mathrm{~B}$. One unit of $\mathrm{A}$ is equivalent to $\mathrm{R}_{\mathrm{i}}$ units of B.: 


$$
R_{i}=\frac{d_{B_{i}}}{d_{A_{i}}}=\frac{\left(\frac{i}{n+1}\right) D_{B}}{\left(1-\frac{i}{n+1}\right) D_{A}}=\frac{i D_{B}}{(n+1-i) D_{A}}, 1 \leq i \leq n
$$

Where:

$\mathrm{n}=$ number of mixtures to be tested

$\mathrm{i}=$ ratio identifier $(\mathrm{i}=1, \ldots \mathrm{n})$

$\mathrm{D}_{\mathrm{A}}=\mathrm{ECX}$ for pure compound $\mathrm{A}$

$\mathrm{DB}_{\mathrm{B}}=\mathrm{ECX}$ for pure compound $\mathrm{B}$

$\mathrm{d}_{\mathrm{A}_{\mathrm{i}}}=$ amount of compound $\mathrm{A}$ in the $\mathrm{ECx}$ of the mixture for ratio $\mathrm{i}$

$\mathrm{d}_{\mathrm{i}}=$ amount of compound $\mathrm{B}$ in the ECx of the mixture for ratio $\mathrm{i}$

Figure 4 shows a chart with three ratios suggested for testing. The independent action line, connecting the $\mathrm{EC}_{50}$ for Atrazine and CPTA is evenly divided into 4 parts by the ratios selected (with some rounding). Once ratios are identified, the $\mathrm{EC}_{20}, \mathrm{EC}_{50}$ and $\mathrm{EC}_{80}$ for the pure compounds can be used to calculate the amount of A in mixture $\mathrm{R}_{\mathrm{i}}$ that is predicted to give $20 \%$, $50 \%$ and $80 \%$ control, respectively, assuming independent action.

To calculate $\mathrm{d}_{\mathrm{A}}$, amount of $\mathrm{A}$ in mixture $\mathrm{R}_{\mathrm{i}}$, where the mixture gives $\mathrm{x}$ percent control, assuming independent action:

$d_{A}=\frac{s * E C_{X_{A}} * E C_{X_{B}}}{E C_{X_{B}}+R_{i} * E C_{X_{A}}}, 0 \leq R_{i} \leq \infty$

Since:

$\frac{d_{A}}{D_{A}}+\frac{d_{B}}{D_{B}}=s$

To calculate $\mathrm{dB}$, the amount of $\mathrm{B}$ in mixture $\mathrm{R}_{\mathrm{i}}$, where mixture gives $\mathrm{x}$ percent control, assuming independent action:

$$
d_{B}=R_{i} * d_{A}
$$

Figure 5 shows points that can be used to calculate the rates for each of the components of the suggested three fixed ratios. The rates for each of the components are found by dropping vertically and horizontally from these points to the dose axes for CPTA and Atrazine. The three independent action lines connect the $\mathrm{EC}_{20} \mathrm{~s}$, the $\mathrm{EC}_{50} \mathrm{~s}$ and the $\mathrm{EC}_{80} \mathrm{~s}$. Table 2 shows the exact design, with the calculated component rates recommended for three fixed ratios. 
One point of confusion in communicating this methodology to biologists was the requirement for each mixture treatment for a specified ratio that the ratio of the component $\mathrm{A}$ to component B be held constant. The use of the term 'fixed ratio' seemed helpful in reducing this confusion.

Mixture designs were generated for all the weed species. During a planning session with Dr. Gregory Armel we narrowed the design to one ratio per each mixture. This was due to the large number of mixtures that were to be tested. This experiment still required 825 test units due to the number of pure compounds (6), mixtures (5), rates (5), replicates (3) and species (5). The rates to be used for each mixture were selected after reviewing the calculated recommended rates for multiple species. The same design would be run for all 5 species.

Since our design was 'one to many' (five herbicides, each mixed with Atrazine), a dose response for Atrazine was needed that would support all the mixtures. Extra rates were added to allow for a full dose response for the pure compounds and for balance. The generation of the final design was a good exercise in the 'give and take' of effective statistical consulting, beginning with recommended ratios and rates based on the dose response for the pure compounds and ending with a feasible reduced design satisfactory both to the statistician and the biologist. Table 3 shows a subset of the design for the experiment, the rates to be tested for the mixture of Atrazine and CPTA. Both visual responses and fresh weight data were collected for all the experimental units. All the pure compounds were assayed again in the same experiment with the mixtures.

\section{Results and Discussion}

Dose response curves were fit to each of the pure compounds and the mixtures and the isobolograms generated. Analyses were done on both the visual response data and the fresh weight data. Isobolograms for the $\mathrm{EC}_{50}$ were generated by parsing the $\mathrm{EC}_{50}$ 's for the mixtures into the mixture components using the ratio tested.

\subsection{Results showing antagonism}

Table 4 shows the results for the mixture of 10 parts CPTA to 1 part Atrazine on morningglory. The table shows the average percent control (based on visual rating) and the ECx estimates. Table 5 shows the confidence intervals for the EC50 for the mixture 10 parts CPTA to 1 part Atrazine on morningglory used to create the confidence intervals on the mixture line. Figure 6 shows these results plotted in an isobologram. Since the entire confidence interval for the 10: 1 ratio isobologram is above the independent action line, antagonism is indicated. More of the compound was required to yield 50\% control than was expected under the assumption of independent action.

\subsection{Results showing Synergy}

Table 6 shows both the visually assessed percent control and the fresh weight results for the same mixture, 10 CPTA: 1 Atrazine, but for a different weed species, common cocklebur. The results for cocklebur for the same mixture of CPTA and Atrazine showed synergy, for both 
visual and fresh weight responses. In both figures 7 and 8, the entire confidence interval for the mixture is below independent action line, indicating synergy. Less of the mixture was required to yield 50\% control than was expected under the assumption of independent action.

\subsection{Using Isobole Method where One Compound has No Activity}

Even if one of the compounds tested has no activity on the species tested, the isobole method can be used to assess results for a mixture. Table 7 shows percent control results for the mixture of 4 parts Norflurazon to 1 part of Atrazine on broadleaf signalgrass. Even though Atrazine has no activity, synergy or antagonism for the mixture can still be assessed. Figure 9 shows a horizontal independent action line, since Atrazine alone was inactive. Under the assumption of independent action, no matter how much Atrazine is added, the amount of Norflurazon needed to give $50 \%$ control does not change. Since the entire confidence interval for the 4:1 mixture falls above the independent action line, the conclusion is antagonism. The red dotted lines are the confidence intervals for Norflurazon's $E_{50}$ of 875: $(753,1014)$.

\section{Summary}

The Colby approach to assessing interaction in mixtures is easy to calculate and interpret. The isobole method, though not as simple, has the advantage of being based on the dose response of the pure compounds and the mixtures. In crop protection studies, dose response assays effectively characterize the biological activity of a compound. The visualization of the isobologram facilitates communication of results, with a synergistic response yielding a concave up curve under the independent action line and an antagonistic response yielding a concave down curve over the independent action line. Designing a mixture study using the isobole method offers an efficient way to evenly search a mixture space and to get definitive assessments of synergy or antagonism. In the case of the Dr. Gregory Armel's study assessing the differential response of mixtures of the PSII inhibitor atrazine with bleaching herbicides, in one well-planned experiment, definitive assessments of synergy and antagonism were produced.

\section{Acknowledgements}

Thanks to Dr. Gregory Armel and the other members of their DuPont Crop Protection Global Technology team (M.C. McComrick, P.L. Rardon, D.A. Baxter, L. Doricchi, L.F. Houck, T.P. Blaesser, B. Kamireddy, R. Kucharczyk, V. Wittenbach, S. Lee) for using the above mentioned isobole method in the design and analysis for their study of the 'Differential response of atrazine mixtures with bleaching herbicides that target different sites in carotenoid biosynthesis and sharing their results.

\section{References}

Berenbaum, M.C. 1989. What is synergy. Pharmacological Reviews Vol. 1989 (41): 93-141.

Colby, S. R. "Calculating Synergistic and Antagonistic Responses of Herbicide Combinations," Weeds, 15(1), pp 20-22 (1967)).” 
Armel, G.R., M.C. McComrick, P.L. Rardon, D.A. Baxter, L. Doricchi, N.M. Ferry, L.F. Houck, T.P. Blaesser, B. Kamireddy, R. Kucharczyk, V. Wittenbach, S. Lee. 2005. Differential response of atrazine mixtures with bleaching herbicides that target different sites in carotenoid biosynthesis. Weed Science Society of America meeting. Soc. Am. Abstr. 45:239.

Figures and Tables

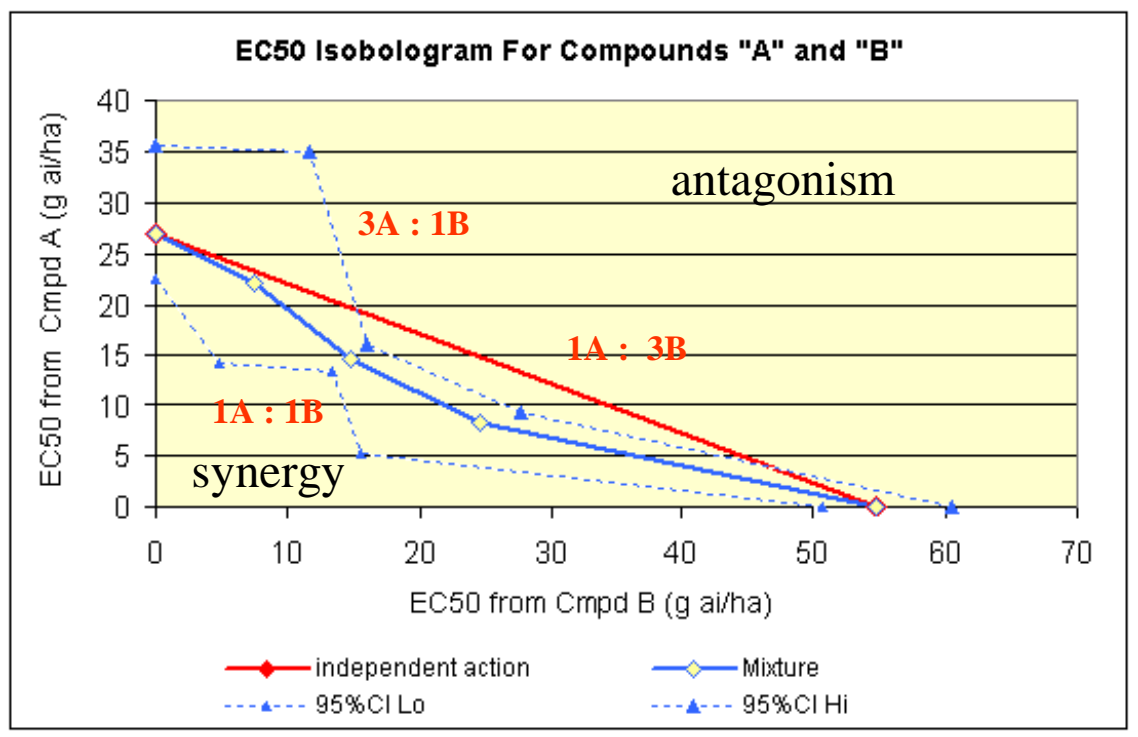

Figure 1. Isobologram for three fixed-ratio mixtures. If the upper confidence interval for a mixture falls under the independent action line, synergy is indicated. If the lower confidence interval for a mixture falls above the independent action line, antagonism is indicated. Mixtures with ratios $1 \mathrm{~A}: 1 \mathrm{~B}$ and $1 \mathrm{~A}: 3 \mathrm{~B}$ show synergy. The mixture with ratio 3A:1B shows neither synergy nor antagonism, as the confidence interval covers the independent action line. 


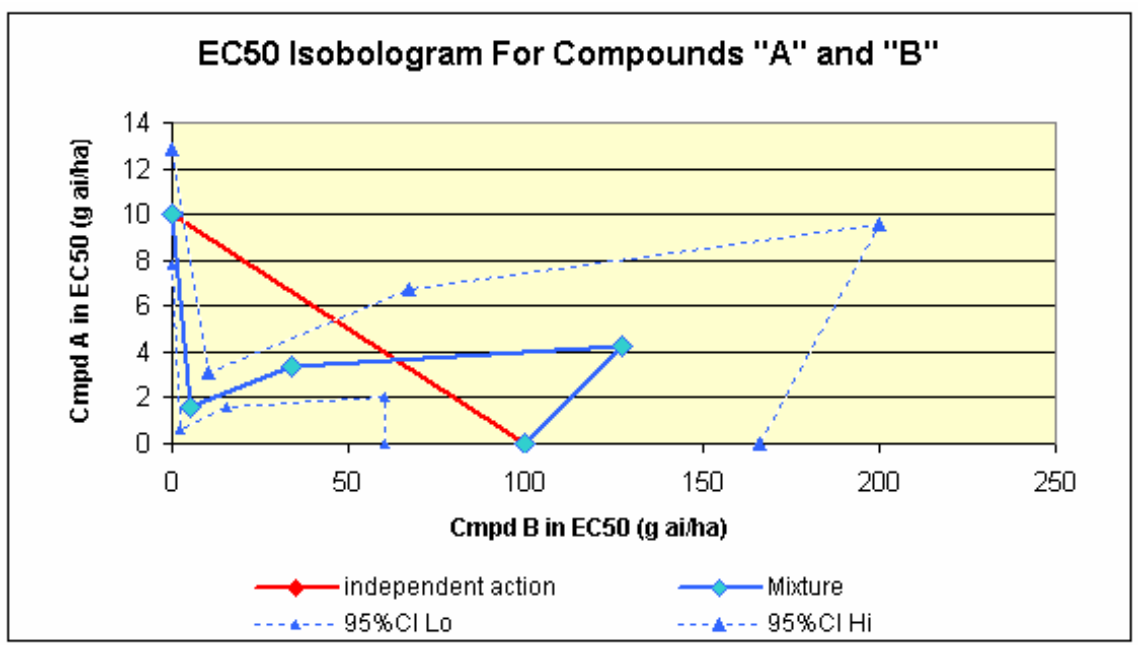

Figure 2. Varying ratios of the same two components can produce results of synergy and possible antagonism. This example points to the need to investigate your mixture space.
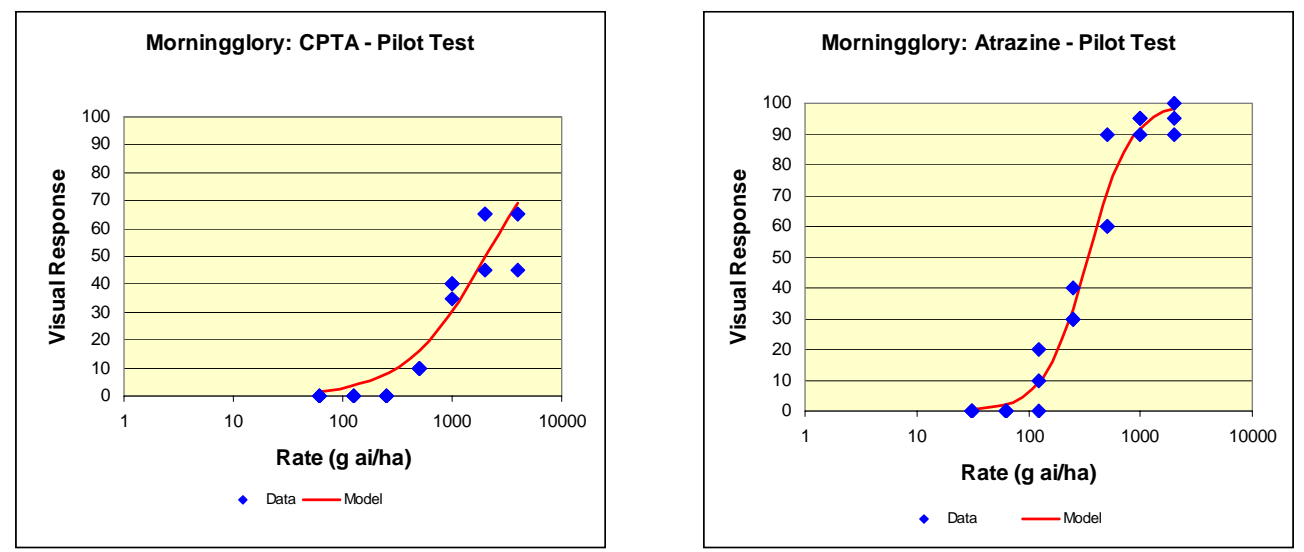

Figure 3. Dose response curves fit to the data (visual assessment of percent control) from the pilot test for two of the pure compounds, CPTA and Atrazine on morningglory. 


\begin{tabular}{|r|r|}
\hline \multicolumn{2}{|c|}{ CPTA on MG } \\
\hline Rate(g/ha) & Avg Resp \\
\hline 62 & 0 \\
\hline 125 & 0 \\
\hline 250 & 0 \\
\hline 500 & 10 \\
\hline 1000 & 38 \\
\hline 2000 & 58 \\
\hline 4000 & 58 \\
\hline \hline EC20 & 624 \\
\hline EC50 & 2021 \\
\hline EC80 & 6545 \\
\hline
\end{tabular}

\begin{tabular}{|r|r|}
\hline \multicolumn{2}{|c|}{ Atrazine on MG } \\
\hline Rate(g/ha) & Avg Resp \\
\hline 31 & 0 \\
\hline 62 & 0 \\
\hline 125 & 10 \\
\hline 250 & 33 \\
\hline 500 & 70 \\
\hline 1000 & 93 \\
\hline 2000 & 95 \\
\hline \hline EC20 & 184 \\
\hline EC50 & 341 \\
\hline EC80 & 630 \\
\hline
\end{tabular}

Table 1: Data summary tables and $\mathrm{EC}_{\mathrm{X}}$ estimates from pilot test for compounds shown in figure 3.

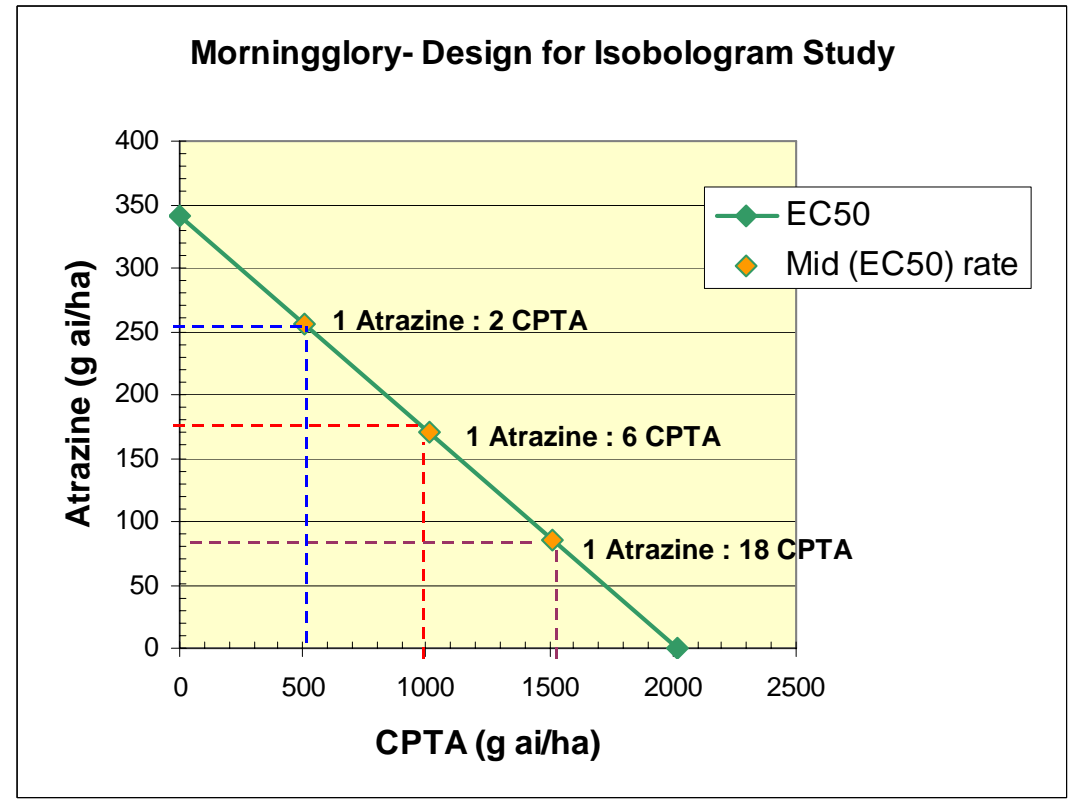

Figure 4. Three ratios suggested for assessing mixture of Atrazine and CPTA on morningglory. The independent action line, connecting the $\mathrm{EC}_{50}$ for Atrazine and CPTA is evenly divided into 4 parts by the three ratios selected (with some rounding). 


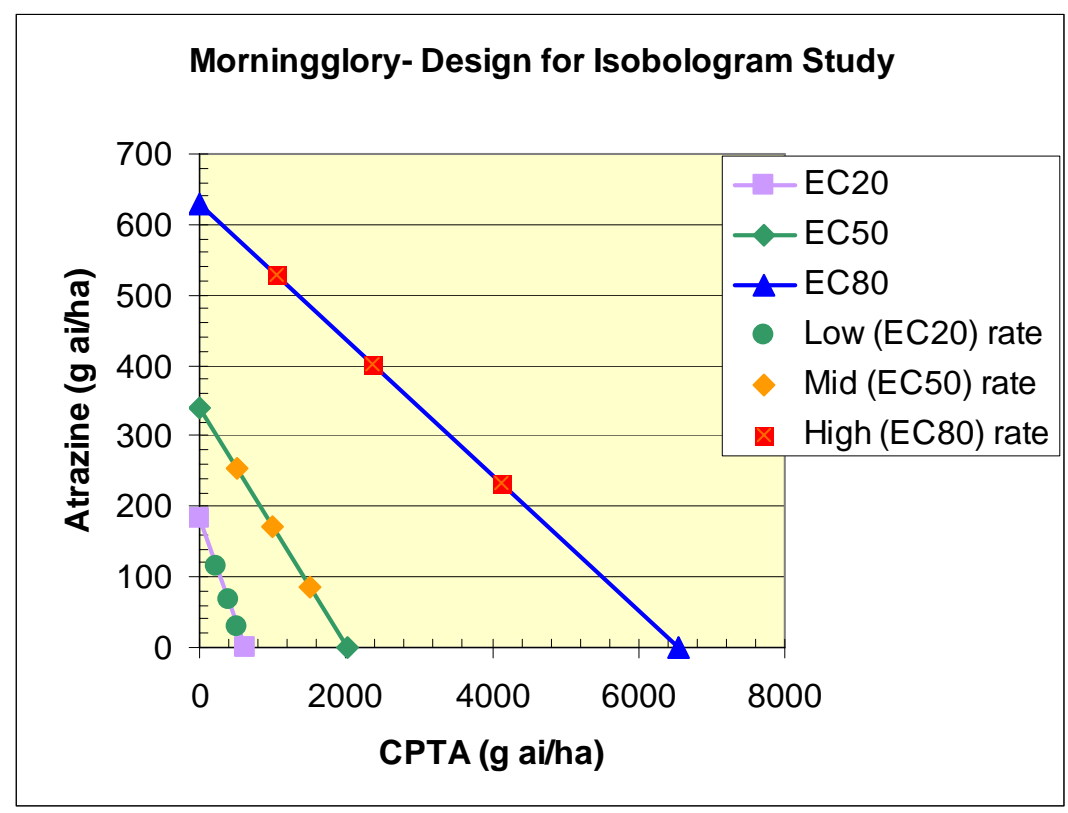

Figure 5 shows points that can be used to calculate the rates for each of the components of the suggested three fixed ratios. The three independent action lines connect the $\mathrm{EC}_{20} \mathrm{~S}$, the $\mathrm{EC}_{50} \mathrm{~S}$ and the $\mathrm{EC}_{80} \mathrm{~s}$.

\begin{tabular}{|c|c|c|c|}
\hline \multicolumn{3}{|c|}{ Recommended Mixture Design } & \\
\hline Species: & compound A: & Atrazine & \\
\hline Morningglory & compound $\mathrm{B}$ : & CPTA & \\
\hline For Ratio (A:B) & $1: 1.98$ & & \\
\hline & Amt of $\mathrm{A}$ & Amt of B & Total g ai \\
\hline dose $\mathrm{EC} 20$ & 116 & 230 & $\begin{array}{r}347 \\
\end{array}$ \\
\hline dose $\mathrm{EC} 50$ & 255 & 506 & 761 \\
\hline dose $\mathrm{ECBO}$ & 529 & 1047 & 1576 \\
\hline For Ratio (A:B) & $1: 5.93$ & & \\
\hline & Amt of A & Amt of B & Total g ai \\
\hline dose $\mathrm{EC} 20$ & 67 & 397 & 464 \\
\hline dose $\mathrm{EC50}$ & 170 & 1010 & 1181 \\
\hline dose $\mathrm{ECBO}$ & 401 & 2377 & 2778 \\
\hline For Ratio (A:B) & $1: 17.8$ & & \\
\hline & Amt of A & Amt of B & Total g ai \\
\hline dose $\mathrm{EC} 20$ & 29 & 524 & 554 \\
\hline dose $\mathrm{EC50}$ & 85 & 1516 & 1601 \\
\hline dose $\mathrm{ECBO}$ & 232 & 4132 & 4364 \\
\hline
\end{tabular}


Table 2. The three ratios calculated for the mixture of Atrazine and CPTA with the rates recommended to yield 20\%, 50\% and $80 \%$ control, under the assumption of independent action.

\begin{tabular}{|c|c|c|c|c|c|c|c|}
\hline \# of pures & \# of rates & \# of reps & \# of pots & \multicolumn{4}{|c|}{ Needed this plus yellow for EC50s for pures } \\
\hline 6 & 5 & 3 & 90 & \multicolumn{4}{|c|}{ Needed to get EC50s for mixtures } \\
\hline & & & & \multicolumn{4}{|c|}{ Needed for balance } \\
\hline \multicolumn{2}{|c|}{ \# of mixtures \# of rates } & $\#$ of reps & \# of pots & & & & \\
\hline 5 & 5 & 3 & 75 & & & & \\
\hline \multicolumn{3}{|c|}{ Total pots for one species } & 165 & & & & \\
\hline \multicolumn{3}{|c|}{ Total pots for five species } & 825 & & & & \\
\hline \multicolumn{2}{|c|}{ Atrazine alone } & \multicolumn{2}{|l|}{ CPTA alone } & \multicolumn{4}{|c|}{ For Ratio: 1 part Atrazine : 10 parts CPTA } \\
\hline & & & & & Atrazine & CPTA & Total g ai \\
\hline 62 & & 625 & & & 62.5 & 625 & \\
\hline 125 & & 1250 & & dose $\mathrm{EC20}$ & 125 & 1250 & 1375 \\
\hline 250 & & 2500 & & dose EC50 & 250 & 2500 & 2750 \\
\hline 500 & & 5000 & & dose EC80 & 500 & 5000 & 5500 \\
\hline 1000 & & 10000 & & & 1000 & 10000 & \\
\hline
\end{tabular}

Table 3: Final design used for mixture of Atrazine with CPTA.

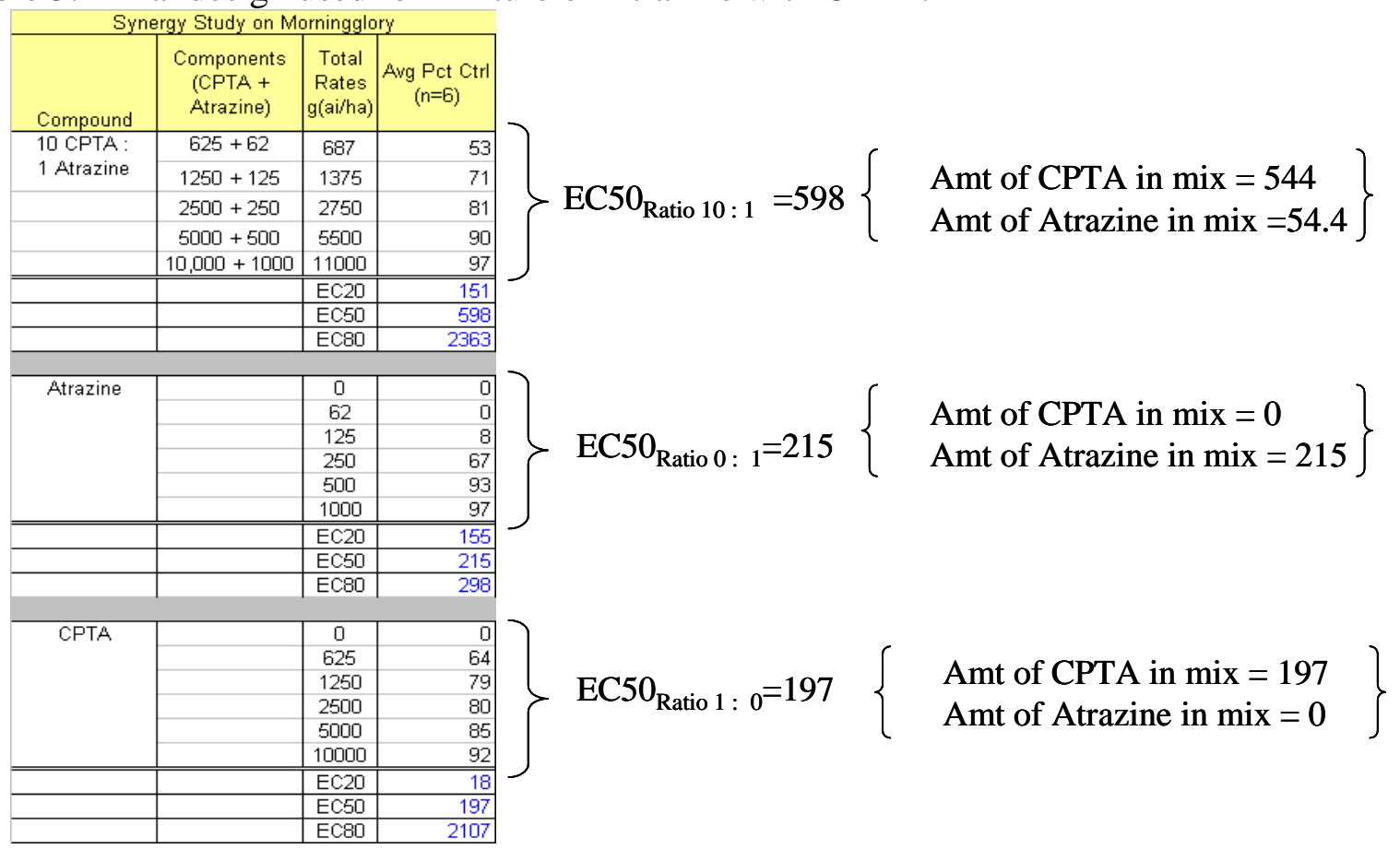

Table 4 shows the results for the mixture of 10 CPTA : 1 Atrazine on morningglory. The table shows the average percent control (based on visual rating) and the $\mathrm{EC}_{\mathrm{X}}$ estimates. The $\mathrm{EC}_{50}$ estimate for the mixture is parsed into the amount of Atrazine in the $\mathrm{EC}_{50}$ and the amount of CPTA in the $\mathrm{EC}_{50}$, using the ratio. 


\begin{tabular}{|c|c|c|c|}
\hline MG & Cmpd A & Cmpd B & Mixture \\
\hline & CPTA & Atrazine & CPTA/Atrazine \\
\hline EC50 & 197.30 & 214.99 & 598.10 \\
\hline Lower & 21.20 & 201.19 & 435.58 \\
\hline Upper & 421.61 & 227.60 & 742.53 \\
\hline Ratio (A:B) & $1: 0$ & $0: 1$ & $10: 1$ \\
\hline
\end{tabular}

Table 5 shows the confidence intervals for the $\mathrm{EC}_{50}$ for the mixture 10 CPTA : 1 Atrazine on morningglory. The standard errors (and thus confidence intervals for the ECx's) were estimated using the Fisher Information Matrix, rather than the inverse Hessian matrix, so they may differ from those given by other programs such as SAS. These confidence intervals are also parsed into the amount due to CPTA and the amount due to Atrazine and are used to create confidence intervals on the mixtures line in the isobologram.

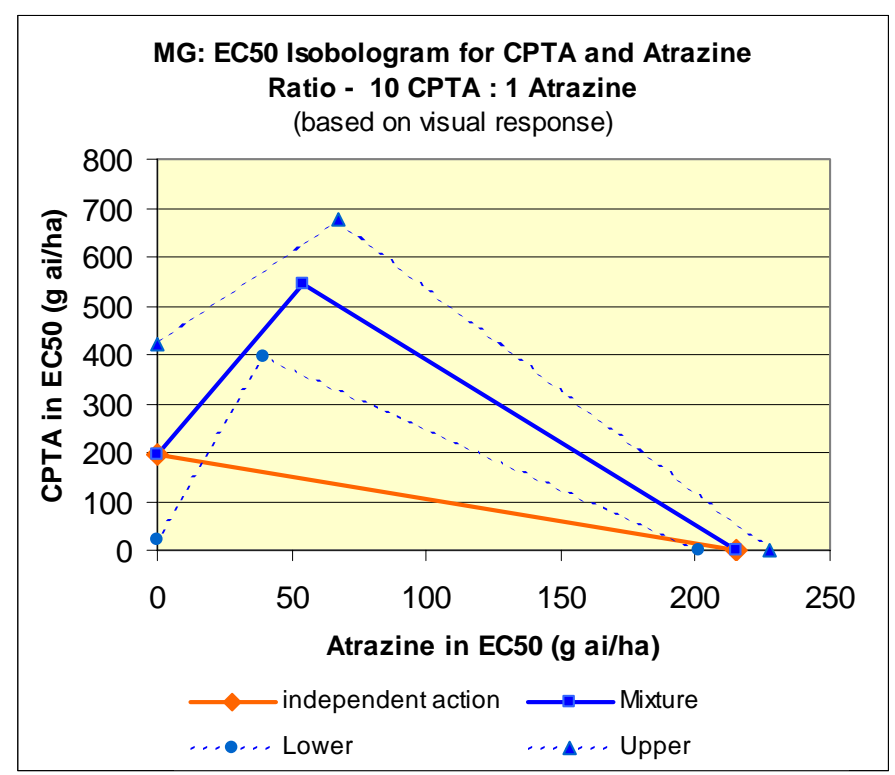

Figure 6 shows isobologram for mixture 10 CPTA: 1 Atrazine on morningglory. Since the entire confidence interval for the mixture line is above the independent action line, antagonism is indicated. More of the mixture was required to yield 50\% control than was expected under the assumption of independent action. 


\begin{tabular}{|c|c|c|r|r|}
\hline \multicolumn{5}{|c|}{ Synergy Study on Common Cocklebur (n=6 reps) } \\
\hline & $\begin{array}{c}\text { Components } \\
\text { (CPTA }+ \\
\text { Atrazine) }\end{array}$ & $\begin{array}{c}\text { Total } \\
\text { Rates } \\
\text { g(ai/ha) }\end{array}$ & $\begin{array}{c}\text { Avg } \\
\text { Pct Ctrl }\end{array}$ & $\begin{array}{c}\text { Avg Fresh } \\
\text { Weight } \\
\text { (grams) }\end{array}$ \\
\hline 10 CPTA : & $0+0$ & 0 & 0 & 5.80 \\
\cline { 2 - 5 } A.trazine & $625+62$ & 687 & 53 & 2.12 \\
\hline & $1250+125$ & 1375 & 68 & 1.31 \\
\hline & $2500+250$ & 2750 & 88 & 0.61 \\
\hline & $5000+500$ & 5500 & 98 & 0.45 \\
\hline & $10,000+1000$ & 11000 & 100 & 0.15 \\
\hline & & EC20 & 248 & 108 \\
\hline & & EC50 & 681 & 408 \\
\hline & & & 1868 & 1538 \\
\hline Synergy & & & Synergy & Synergy \\
\hline Assessment & & & &
\end{tabular}

Table 6. Results for mixture of 10 CPTA :1 Atrazine on common cocklebur. Table gives average percent control based on visual assessment, average fresh weight and estimated $\mathrm{EC}_{\mathrm{X}}$ 's. 

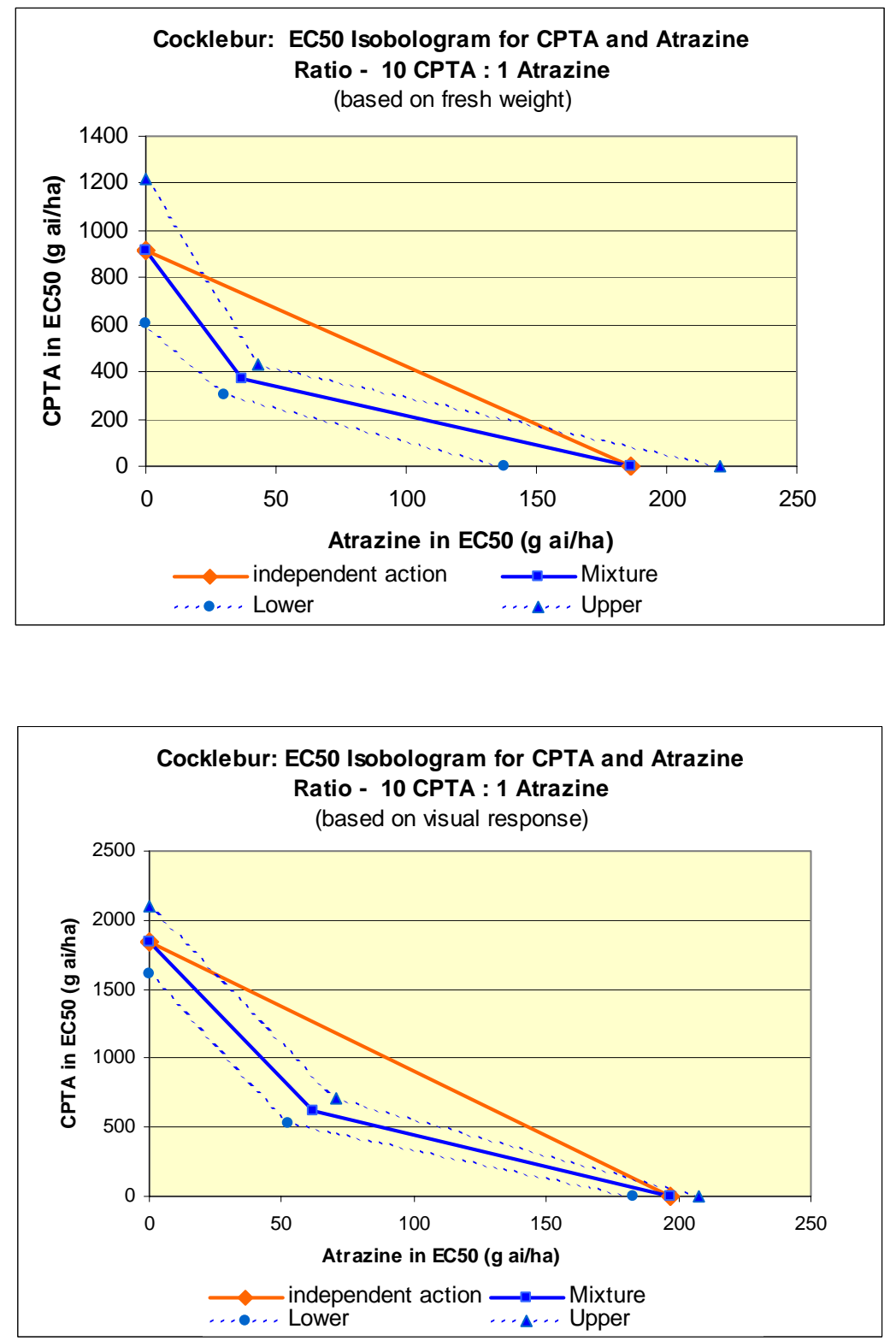

Figures 7 and 8. Results based on fresh weight and visual assessment of percent control for the mixture 10 CPTA : 1Atrazine on cocklebur. Both isobolograms show synergy, since in both cases the entire confidence interval around the mixture line is below independent action line. Less of the mixture was required to yield 50\% control than was expected under the assumption of independent action. 


\begin{tabular}{|c|c|c|c|c|c|c|}
\hline \multicolumn{7}{|c|}{ Synergy Study on Broadleaf Signalgrass ( $n=6$ reps) } \\
\hline \multicolumn{3}{|c|}{4 Norflurazon: 1 Atrazine } & \multicolumn{2}{|c|}{ Atrazine } & \multicolumn{2}{|c|}{ Norflurazon } \\
\hline $\begin{array}{l}\text { Components } \\
\text { (4 Norflurazon } \\
+1 \text { Atrazine) }\end{array}$ & $\begin{array}{c}\text { Total } \\
\text { Rates } \\
\text { g(ai/ha) }\end{array}$ & $\begin{array}{c}\text { Avg } \\
\text { Pct Ctrl }\end{array}$ & $\begin{array}{c}\text { Atrazine } \\
\text { Rate } \\
\text { g(ai/ha) }\end{array}$ & $\begin{array}{c}\text { Avg } \\
\text { Pct Ctrl }\end{array}$ & $\begin{array}{c}\text { Norflurazon } \\
\text { Rate } \\
\text { g(ai/ha) }\end{array}$ & $\begin{array}{c}\text { Avg } \\
\text { Pct Ctrl }\end{array}$ \\
\hline $250+62$ & 312 & 11 & 62 & 0 & 250 & 13 \\
\hline $500+125$ & 625 & 14 & 125 & 0 & 500 & 22 \\
\hline $1000+250$ & 1250 & 41 & 250 & 0 & 1000 & 66 \\
\hline $2000+500$ & 2500 & 73 & 500 & 0 & 2000 & 75 \\
\hline \multirow{5}{*}{$4000+1000$} & 5000 & 85 & 1000 & 11 & 4000 & 85 \\
\hline & EC20 & 680 & EC20 & 1039 & EC20 & 356 \\
\hline & EC50 & 1527 & EC50 & & EC50 & 875 \\
\hline & EC80 & 3429 & EC80 & & EC80 & 2151 \\
\hline & & Antagonism & & no dose resp & & \\
\hline
\end{tabular}

Table 7. Results for mixture of 4 Norflurazon : 1 Atrazine on broadleaf signalgrass. Even though Atrazine has no activity, synergy or antagonism for the mixture can still be assessed.

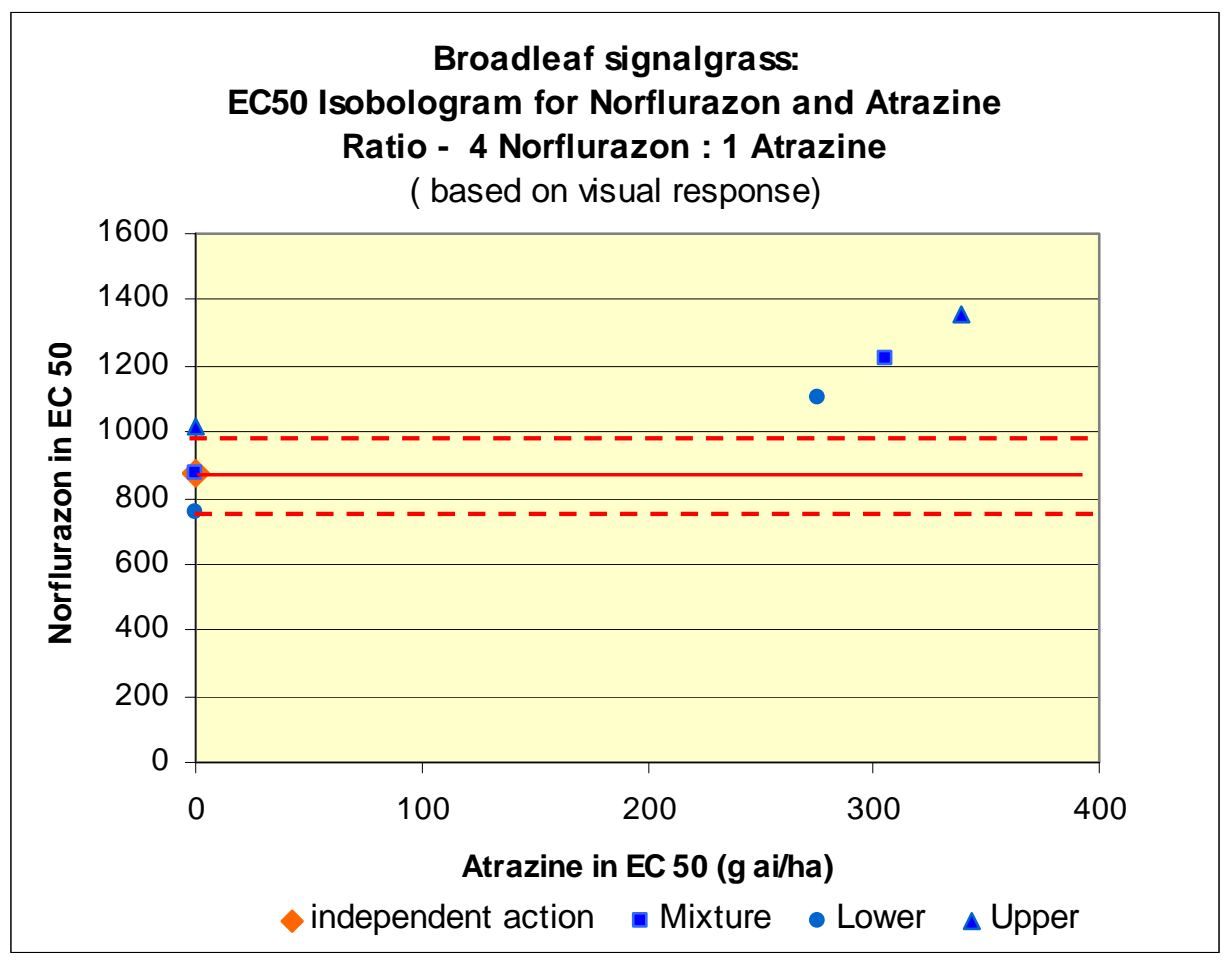

Figure 9. There is a horizontal independent action line, since Atrazine alone was inactive. Under the assumption of independent action, no matter how much Atrazine is added, the amount of Norflurazon needed to give 50\% control does not change. Since the entire confidence interval for the 4:1 mixture falls above the independent action line, the conclusion is antagonism. The red dotted lines are the confidence intervals for Norflurazon's EC Fo $_{50}$ of : $(753,1014)$. 